From Amgen Inc, Thousand Oaks, CA; Ghent University Hospital, Ghent,

Belgium; University Hospital Gasthuisberg, Leuven, Belgium; and the Ospedale Niguarda Ca' Granda, Milan, Italy.

Submitted October 1, 2007; accepted November 20, 2007; published online ahead of print at www.jco.org on March 3, 2008.

Funded by Amgen Inc, Thousand Oaks, CA

Presented in part in oral format at the 14th European Cancer Conference, Barcelona, Spain, September 23-27, 2007; and the American Society of Clinical Oncology Gastrointestinal Cancer Symosium, Orlando, FL January 25-27, 2008

Authors' disclosures of potential conflicts of interest and author contributions are found at the end of this article.

Corresponding author: Rafael G. Amado, MD, Amgen, Inc, One Amgen Center Dr, MS 38-2-B, Thousand Oaks, CA 91320-1799; e-mail: ramado@ amgen.com.

(C) 2008 by American Society of Clinical Oncology

0732-183X/08/2610-1626/\$20.00 DOI: 10.1200/JCO.2007.14.7116

\title{
Wild-Type KRAS Is Required for Panitumumab Efficacy in Patients With Metastatic Colorectal Cancer
}

Rafael G. Amado, Michael Wolf, Marc Peeters, Eric Van Cutsem, Salvatore Siena, Daniel J. Freeman, Todd Juan, Robert Sikorski, Sid Suggs, Robert Radinsky, Scott D. Patterson, and David D. Chang

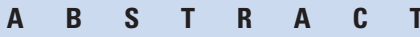

\section{Purpose}

Panitumumab, a fully human antibody against the epidermal growth factor receptor (EGFR), has activity in a subset of patients with metastatic colorectal cancer (mCRC). Although activating mutations in KRAS, a small G-protein downstream of EGFR, correlate with poor response to anti-EGFR antibodies in $\mathrm{mCRC}$, their role as a selection marker has not been established in randomized trials.

\section{Patients and Methods}

KRAS mutations were detected using polymerase chain reaction on DNA from tumor sections collected in a phase III mCRC trial comparing panitumumab monotherapy to best supportive care (BSC). We tested whether the effect of panitumumab on progression-free survival (PFS) differed by KRAS status.

\section{Results}

KRAS status was ascertained in 427 (92\%) of 463 patients (208 panitumumab, 219 BSC). KRAS mutations were found in $43 \%$ of patients. The treatment effect on PFS in the wild-type (WT) KRAS group (hazard ratio [HR], $0.45 ; 95 \% \mathrm{Cl}: 0.34$ to 0.59$)$ was significantly greater $(P<.0001)$ than in the mutant group (HR, 0.99; 95\% Cl, 0.73 to 1.36). Median PFS in the WT KRAS group was 12.3 weeks for panitumumab and 7.3 weeks for BSC. Response rates to panitumumab were $17 \%$ and $0 \%$, for the WT and mutant groups, respectively. WT KRAS patients had longer overall survival (HR, 0.67; 95\% Cl, 0.55 to 0.82; treatment arms combined). Consistent with longer exposure, more grade III treatment-related toxicities occurred in the WT KRAS group. No significant differences in toxicity were observed between the WT KRAS group and the overall population.

\section{Conclusion}

Panitumumab monotherapy efficacy in $\mathrm{mCRC}$ is confined to patients with WT KRAS tumors. KRAS status should be considered in selecting patients with $\mathrm{mCRC}$ as candidates for panitumumab monotherapy.

\section{J Clin Oncol 26:1626-1634. (c) 2008 by American Society of Clinical Oncology}

\section{INTRODUCTION}

Epidermal growth factor receptor (EGFR) has been validated as a therapeutic target in several human tumors, including colorectal cancer (CRC). ${ }^{1-4} \mathrm{Li}$ gand occupancy of the EGFR activates the RAS/ RAF/MAPK, STAT, and PI3K/AKT signaling pathways, which together modulate cellular proliferation, adhesion, angiogenesis, migration, and survival. $^{5,6}$ The anti-EGFR targeted antibodies cetuximab and panitumumab administered as monotherapy in CRC have shown response and disease stabilization rates of approximately $10 \%$ and $30 \%$, respectively. ${ }^{2,3}$ Although EGFR expression is used for patient selection, clinical experience shows that the level of EGFR expression as measured by immunohistochemistry does not predict clinical benefit. ${ }^{2,7-9}$

KRAS, the human homolog of the Kirsten rat sarcoma-2 virus oncogene, encodes a small GTPbinding protein that acts as a self-inactivating signal transducer by cycling from GDP- to GTP-bound states in response to stimulation of a cell surface receptor, including EGFR. ${ }^{10,11}$ KRAS can harbor oncogenic mutations that yield a constitutively active protein. ${ }^{10-13}$ Such mutations are found in approximately $30 \%$ to $50 \%$ of CRC tumors and are common in other tumor types. ${ }^{12,14-19}$ Several studies have indicated that the presence of mutant KRAS in lung and CRC tumors correlates with poor prognosis, ${ }^{14,17,18,20}$ and is associated with lack of response to EGFR inhibitors. ${ }^{15,16,19,21,22}$ These published reports investigating the role of KRAS as a selection 
marker for EGFR inhibitor treatment were based on tumor samples from uncontrolled studies and included patients treated with antiEGFR antibodies alone or in combination with irinotecan. Given the possible prognostic role of KRAS mutational status, these uncontrolled studies could not isolate the relative effect of antibody treatment on outcome by KRAS status from the prognostic implications of KRAS as a marker of poor clinical outcome in CRC.

We assessed the predictive role of KRAS in a phase III, randomized trial comparing panitumumab monotherapy with best supportive care (BSC) in patients with chemotherapy-refractory metastatic CRC. ${ }^{3}$ The primary objective of the biomarker analyses was to determine whether the effect of panitumumab monotherapy on progression-free survival (PFS) differed between patients whose tumors contain mutant versus wild-type (WT; ie, nonmutated) KRAS.

\section{PATIENTS AND METHODS}

\section{Trial Design and Patient Population}

The design of this controlled, panitumumab monotherapy study has been previously described. ${ }^{3}$ Briefly, patients with metastatic CRC with EGFR expression in $\geq 1 \%$ of tumor cells (assessed by immunohistochemistry) and documented evidence of disease progression after failure of fluoropyrimidines and prespecified exposure to oxaliplatin and irinotecan were randomly assigned to panitumumab $6 \mathrm{mg} / \mathrm{kg}$ plus BSC every 2 weeks or BSC alone. BSC patients could receive panitumumab after disease progression. Tumor status was assessed radiographically every 4 to 8 weeks from week 8 until disease progression using the Response Evaluation Criteria in Solid Tumors by blinded central review. The primary end point was PFS, defined as the interval from random assignment to radiologic progression or death. Secondary end points included objective response rate, overall survival (OS), and safety. All patients, including those with unassessable or missing assessments, were included in the response rate analysis. A best response of stable disease was determined at or after week 8 from random assignment. At enrollment, patients provided informed consent for study procedures including research on archived paraffin-embedded tumor samples (mostly from primary tumor resection) for identification of predictive biomarkers. The study protocol was approved by the ethics board at each research center.

\section{Assay to Detect Mutant KRAS}

Formalin-fixed, paraffin-embedded tumor sections were deparaffinized and air dried, and DNA was isolated using proteinase K and a DNeasy minispin column (Qiagen, Valencia, CA). Mutant KRAS was detected using a validated KRAS mutation kit (DxS Ltd, Manchester, United Kingdom) that identifies seven somatic mutations located in codons 12 and 13 (Gly12Asp, Gly12Ala, Gly12Val, Gly12Ser, Gly12Arg, Gly12Cys, and Gly13Asp) using allele-specific real-time polymerase chain reaction. ${ }^{23-25}$ A central laboratory (HistoGeneX, Antwerp, Belgium) validated the assay for analytic and diagnostic performance, established acceptance criteria, included appropriate quality controls for each assay, and performed the KRAS analysis in a blinded fashion.

\section{Statistical Analysis}

The primary objective of the biomarker analyses was to examine whether the relative effect of panitumumab compared with BSC on PFS differed in patients with tumors bearing mutant versus WT KRAS. Additional objectives included examining whether panitumumab improved PFS, OS, and response rate in the WT KRAS group compared with the BSC group. Safety was assessed in both KRAS groups. Analyses were limited to patients with known KRAS status and were categorized by randomized treatment for efficacy and safety. Adverse events were graded per the National Cancer Institute Common Toxicity Criteria version 2.0 with the exception of selected skin toxicities, which were graded using version 3.0. Statistical analyses were performed at Amgen Inc. All analyses were prespecified in a statistical analysis plan before KRAS mutation assessment.
A quantitative-interaction test ${ }^{26}$ at a two-sided $5 \%$ level was used to compare the PFS log-hazard ratio (HR; panitumumab relative to BSC) from a Cox model with covariates for the randomization factors between the WT and mutant KRAS groups. Based on an assessable sample size of 380 patients and assuming $60 \%$ WT prevalence, power was estimated at more than $99 \%$ if the HR was 1.0 in the mutant KRAS group and at $87 \%$ if the HR was 0.80 in the mutant KRAS group, assuming an overall HR of 0.54 among all patients. Kaplan-Meier methods were used to estimate PFS and OS. Conditional on a significant interaction test, sequential testing at a $5 \%$ level of PFS, followed by OS and overall response rate, were planned within the WT KRAS group between panitumumab versus BSC. A log-rank test was used for PFS, Wilcoxon for OS, and a generalized Cochran-Mantel-Haenszel test for response rate, each stratified by the randomization factors.

Maximum change in tumor burden per blinded central radiology review was summarized by treatment in each KRAS group. Propensity-score sensitivity analyses were performed to assess bias due to exclusion of patients with unknown KRAS status.

\section{RESULTS}

\section{Patients}

Of the 463 patients originally enrolled, ${ }^{3} 427$ (92\%) were included in the KRAS analyses (208 and 219 in the panitumumab and BSC arms, respectively; Fig 1). KRAS status could not be determined in 18 patients because of unavailable samples and in an additional 18 patients whose samples had insufficient or poor-quality DNA. KRAS mutations were identified in $184(43 \%)$ of 427 patients (84 [40\%] and $100[46 \%]$ in the panitumumab and BSC arms, respectively). In the BSC arm, 76\% of patients with WT KRAS and 77\% of patients with mutant KRAS received panitumumab in a cross-over protocol, after a median PFS time in the original study (investigator assessment) of 7.1 weeks (95\% CI, 7.0 to 7.6) and 6.3 weeks (95\% CI, 5.1 to 7.1) for patients in the WT and mutant KRAS groups, respectively.

Baseline patient characteristics were balanced between the WT and mutant KRAS groups for both panitumumab and BSC (Table 1). The distribution of specific KRAS mutations was similar between treatment arms (Table 2).

\section{Efficacy}

Primary end point: PFS. Similar to previously described results in the intent-to-treat population, ${ }^{3}$ a statistically significant improvement in PFS was observed in the KRAS assessable group between panitumumab and BSC (HR, 0.59; 95\% CI, 0.48 to 0.72). Median PFS time was 8.0 weeks for panitumumab and 7.3 weeks for BSC. The relative effect of panitumumab versus BSC on PFS was significantly greater among patients with WT KRAS (HR, 0.45; 95\% CI, 0.34 to 0.59; median PFS of 12.3 weeks for panitumumab $v 7.3$ weeks for BSC) compared with patients with mutant KRAS, in whom no panitumumab benefit was observed (HR, $0.99 ; 95 \%$ CI, 0.73 to 1.36 ; median PFS of 7.4 weeks for panitumumab $v 7.3$ weeks for BSC; Fig 2). The quantitative-interaction test comparing the magnitude of the relative treatment effect on PFS between WT and mutant KRAS groups was statistically significant $(P<.0001)$. Consistent results were obtained with propensity-score adjusted HRs. PFS was significantly greater for panitumumab versus BSC in the WT KRAS group (stratified log-rank test $P<.0001$; Fig 2 ). In all sensitivity analyses performed in the WT KRAS subset, PFS favored the panitumumab arm. In particular, to compensate for potential tumor-ascertainment bias in favor of the BSC arm, an interval-censored sensitivity analysis was performed 


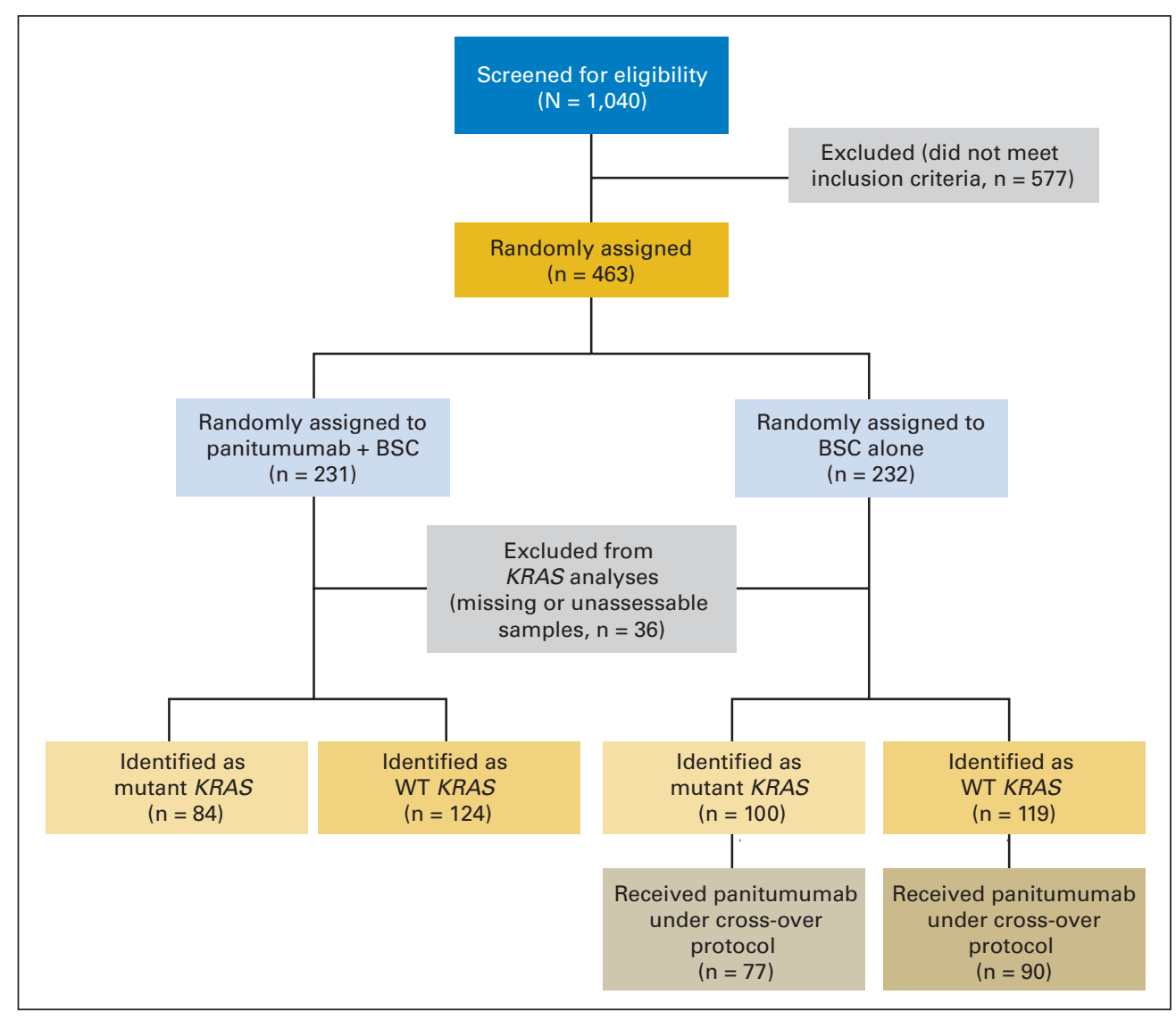

Fig 1. CONSORT diagram. BSC, best supportive care; WT, wild-type.

whereby radiologic event times were moved to the closest assessment time prespecified in the protocol. These analyses showed HR $=0.44$ (95\% CI, 0.30 to 0.63 ) and median PFS times of 16 and 8 weeks for panitumumab and BSC, respectively. Across all subsets examined, the treatment effect of panitumumab on PFS in the WT KRAS group was consistent with the primary analysis (Fig 3). Of 168 BSC patients receiving panitumumab after progression, PFS was significantly longer among patients with WT versus patients with mutant KRAS (HR, 0.32; 95\% CI, 0.22 to 0.45; median PFS time of 16.4 weeks for WT and 7.9 weeks for mutant; online-only Fig A1A).

Response rate. Best overall response data were unassessable or missing for 35 of 231 patients receiving panitumumab and for 53 of 232 BSC patients (this included 16 of 124 patients receiving panitumumab with WT KRAS, 16 of 119 BSC patients with WT KRAS, 15 of 84 patients receiving panitumumab in the mutant KRAS group, and 32 of 100 BSC patients in the mutant KRAS group). In the KRAS assessable group, response rate for panitumumab was $10 \%$, stable disease was $25 \%$, and disease progression was $50 \%$. For KRAS assessable patients in the BSC arm, $0 \%$ had a response, $10 \%$ had stable disease, and $68 \%$ had disease progression. No responders were identified in the panitumumab mutant KRAS group (100\% positive predictive value for nonresponse in the mutant group). In contrast, in the panitumumab WT KRAS group 21 of 124 patients had a partial response (17\%; 95\% CI, $11 \%$ to $25 \%$; Fig 4). Median time to response was 7.9 weeks (range, 7.0 to 15.6 weeks), and median duration of response was 19.7 weeks (range, 7.9 to 88.7 weeks).

In the WT KRAS group, 42 patients receiving panitumumab (34\%) and 14 BSC patients (12\%) had stable disease (Fig 4). In the mutant KRAS group, stable disease was observed in $10(12 \%)$ and eight patients $(8 \%)$ in the panitumumab and BSC arms, respectively. Consistent results with PFS and response were observed when examining the magnitude of effect on target lesions for individual patients. For the WT KRAS group, $61 \%$ of patients receiving panitumumab with available target lesion measurements (62 of 101 in the WT group) had a target lesion decrease, including the majority of patients with stable disease (Fig 4). In contrast, in the mutant KRAS group, only 5\% of patients receiving panitumumab (three of 62) had minor tumor reductions. For the BSC patients in both KRAS groups, $3 \%$ of patients (six of 178) had some degree of tumor reduction.

Of 168 BSC patients in the KRAS assessable group that crossed over to receive panitumumab on progression, 20 (12\%) experienced a response (including one patient with a complete response), and 55 $(33 \%)$ had stable disease. All responders had WT KRAS, for a response rate of 20 of 91 (22\%; 95\% CI, $14 \%$ to $32 \%)$.

OS. At the time of these analyses, a total of 391 KRAS assessable patients (92\%) had died (186 [89\%] patients receiving panitumumab and 205 [94\%] BSC patients). Median follow-up time was 14.1 months for the remaining 36 patients. No statistically significant OS difference was observed between treatment arms among all patients (HR, 0.97; 95\% CI, 0.79 to 1.18), or in either of the KRAS groups; the HR for OS was 1.02 (95\% CI, 0.75 to 1.39 ) and 0.99 (95\% CI, 0.75 to 1.29 ) for the mutant and WT KRAS groups, respectively. OS was longer overall in the WT group than in the mutant group adjusting for stratification factors and randomized treatment (HR, 0.67; 95\% CI, 0.55 to 0.82 ; both arms combined; Fig 5). Multivariate analysis showed that WT KRAS status was a predictor for OS in both the 


\begin{tabular}{|c|c|c|c|c|c|c|c|c|}
\hline \multirow[b]{3}{*}{ Characteristic } & \multicolumn{4}{|c|}{ Mutant } & \multicolumn{4}{|c|}{ Wild-Type } \\
\hline & \multicolumn{2}{|c|}{ Panitumumab } & \multicolumn{2}{|c|}{$\mathrm{BSC}$} & \multicolumn{2}{|c|}{ Panitumumab } & \multicolumn{2}{|c|}{$\mathrm{BSC}$} \\
\hline & No. & $\%$ & No. & $\%$ & No. & $\%$ & No. & $\%$ \\
\hline No. of patients & 84 & & 100 & & 124 & & 119 & \\
\hline \multicolumn{9}{|l|}{ Sex } \\
\hline Male & 47 & 56 & 64 & 64 & 83 & 67 & 76 & 64 \\
\hline \multicolumn{9}{|l|}{ Race/ethnicity } \\
\hline White & 84 & 100 & 97 & 97 & 122 & 98 & 118 & 99 \\
\hline \multicolumn{9}{|l|}{ Baseline age, years } \\
\hline Median & \multicolumn{2}{|c|}{62.0} & \multicolumn{2}{|c|}{62.0} & \multicolumn{2}{|c|}{62.5} & \multicolumn{2}{|c|}{63.0} \\
\hline Minimum & \multicolumn{2}{|c|}{27} & \multicolumn{2}{|c|}{27} & \multicolumn{2}{|c|}{29} & \multicolumn{2}{|c|}{32} \\
\hline Maximum & \multicolumn{2}{|c|}{79} & \multicolumn{2}{|c|}{83} & \multicolumn{2}{|c|}{82} & \multicolumn{2}{|c|}{81} \\
\hline \multicolumn{9}{|l|}{ Primary diagnosis } \\
\hline Colon cancer & 53 & 63 & 65 & 65 & 86 & 69 & 82 & 69 \\
\hline Rectal cancer & 31 & 37 & 35 & 35 & 38 & 31 & 37 & 31 \\
\hline \multicolumn{9}{|l|}{ ECOG performance status } \\
\hline 0 & 43 & 51 & 37 & 37 & 53 & 43 & 40 & 34 \\
\hline 1 & 28 & 33 & 47 & 47 & 56 & 45 & 62 & 52 \\
\hline$\geq 2^{*}$ & 13 & 15 & 16 & 16 & 15 & 12 & 17 & 14 \\
\hline \multicolumn{9}{|c|}{ Cells with EGFR membrane staining } \\
\hline $1 \%$ to $<10 \%$ & 20 & 24 & 23 & 23 & 31 & 25 & 29 & 24 \\
\hline $10 \%$ to $100 \%$ & 63 & 75 & 77 & 77 & 93 & 75 & 89 & 75 \\
\hline \multicolumn{9}{|c|}{ Highest membrane staining intensity } \\
\hline $3+$ (strong) & 17 & 20 & 17 & 17 & 25 & 20 & 22 & 18 \\
\hline $2+$ (moderate) & 42 & 50 & 51 & 51 & 69 & 56 & 58 & 49 \\
\hline $1+($ weak $)$ & 24 & 29 & 32 & 32 & 30 & 24 & 39 & 33 \\
\hline 0 & 1 & 1 & 0 & 0 & 0 & 0 & 0 & 0 \\
\hline \multicolumn{9}{|l|}{ Prior adjuvant chemotherapy } \\
\hline Yes & 27 & 32 & 40 & 40 & 50 & 40 & 32 & 27 \\
\hline \multicolumn{9}{|l|}{ Prior lines of chemotherapy } \\
\hline 2 & 54 & 64 & 74 & 74 & 79 & 64 & 63 & 53 \\
\hline 3 & 23 & 27 & 24 & 24 & 41 & 33 & 49 & 41 \\
\hline
\end{tabular}

panitumumab $(\mathrm{HR}, 0.64 ; P=.004)$ and $\mathrm{BSC}(\mathrm{HR}, 0.68 ; P=.007)$ arms. Similar results for OS were observed among the 168 BSC patients receiving panitumumab after progression $(\mathrm{HR}, 0.65 ; 95 \% \mathrm{CI}$, 0.47 to 0.90 ; median OS time of 6.8 months for WT $v 4.5$ months for mutant; online-only Fig A1B). For the 51 BSC patients who did not cross-over to panitumumab, no difference in OS was observed

\begin{tabular}{|c|c|c|c|c|c|c|}
\hline \multirow{2}{*}{$\begin{array}{c}\text { KRAS } \\
\text { Mutation }\end{array}$} & \multicolumn{2}{|c|}{$\begin{array}{c}\text { Total } \\
(\mathrm{N}=184)\end{array}$} & \multicolumn{2}{|c|}{$\begin{array}{l}\text { Panitumumab } \\
\quad(n=84) \\
\end{array}$} & \multicolumn{2}{|c|}{$\begin{array}{c}\text { BSC } \\
(n=100)\end{array}$} \\
\hline & No. & $\%$ & No. & $\%$ & No. & $\%$ \\
\hline 12Ala & 15 & 8.2 & 8 & 9.5 & 7 & 7.0 \\
\hline 12Asp & $70^{*}$ & 38.0 & 34 & 40.5 & 36 & 36.0 \\
\hline 12Arg & $3^{*}$ & 1.6 & 0 & 0.0 & 3 & 3.0 \\
\hline $12 \mathrm{Val}$ & 40 & 21.7 & 15 & 17.9 & 25 & 25.0 \\
\hline 12 Cys & 14 & 7.6 & 7 & 8.3 & 7 & 7.0 \\
\hline 12Ser & 14 & 7.6 & 5 & 6.0 & 9 & 9.0 \\
\hline 13Asp & 29 & 15.8 & 15 & 17.9 & 14 & 14.0 \\
\hline \multicolumn{7}{|c|}{$\begin{array}{l}\text { Abbreviations: BSC, best supportive care; Ala, alanine; Asp, aspartic acid; } \\
\text { Arg, arginine; Val, valine; Cys, cysteine; Ser, serine. } \\
{ }^{*} \text { Two mutations were detected in one specimen. }\end{array}$} \\
\hline
\end{tabular}

between WT and mutant KRAS groups (median OS time of 1.9 and 2 months, respectively).

\section{Exposure and Safety}

The mean number of panitumumab infusions was 10.0 (median, 8.0) and 4.9 (median, 4.0) in WT and mutant KRAS groups, respectively. In the mutant KRAS group, $100 \%$ of patients receiving panitumumab and $84 \%$ of BSC patients had an adverse event. In the WT KRAS group, these numbers were $100 \%$ and $90 \%$, respectively. By maximum grade and by KRAS group, a higher incidence of grade 3 or 4 adverse events (44\% $v 28 \%$ ) and treatment-related grade 3 adverse events $(25 \% v 12 \%)$ was observed in the panitumumab WT versus mutant KRAS groups, respectively. In the KRAS assessable population, $37 \%$ of patients had a grade 3 or 4 event, and $20 \%$ of patients had a treatment-related grade 3 or 4 adverse events. The incidence of adverse events leading to withdrawal in the panitumumab arm was $7 \%$ and $5 \%$ for the WT and mutant KRAS groups, respectively; $2 \%$ of WT KRAS patients and $1 \%$ of mutant KRAS patients withdrew for panitumumab-related events.

Grade 3 integument-related events occurred in 20\% of all KRAS assessable patients (in 25\% of WT KRAS patients and in $13 \%$ of mutant KRAS patients). In the mutant KRAS group, $1 \%$ of patients 
had a grade 4 integument-related event; there were no grade 4 events in the WT group. The time to any integument-related event or to an event grade 2 or higher was similar in both KRAS groups, suggesting that incidence differences for integument toxicity were due to differential exposure. Consistent with previous reports, ${ }^{2,3}$ patients with the worst grade skin toxicity in the WT KRAS group appeared to experience better PFS and OS (data not shown). In the panitumumab arm, a higher incidence of diarrhea of any grade was observed (WT KRAS $24 \%$; mutant KRAS 19\%) but grade 3 diarrhea was comparable between groups (WT KRAS 2\%; mutant KRAS 1\%). The incidence of hypomagnesemia reported as an adverse event of any grade was 3\% and $0 \%$ for WT and mutant KRAS groups, respectively. One grade 2 infusion reaction was reported as an adverse event in a patient with mutant KRAS.

\section{DISCUSSION}

These results show that KRAS mutations predict for lack of clinical benefit to panitumumab therapy. The presence of a control arm made it possible to study the relative effect of panitumumab monotherapy by KRAS mutational status independent of the potential prognostic influence of KRAS mutations on outcomes, enabling us to conclude that the clinical benefit observed in the KRAS unselected population was entirely derived from the KRAS WT population. Given the crossover design, conclusions are limited to the effect of KRAS mutational status on PFS and tumor response end points and not to OS. Indeed, the majority of BSC patients received panitumumab on disease progression early in the trial in both KRAS groups (median

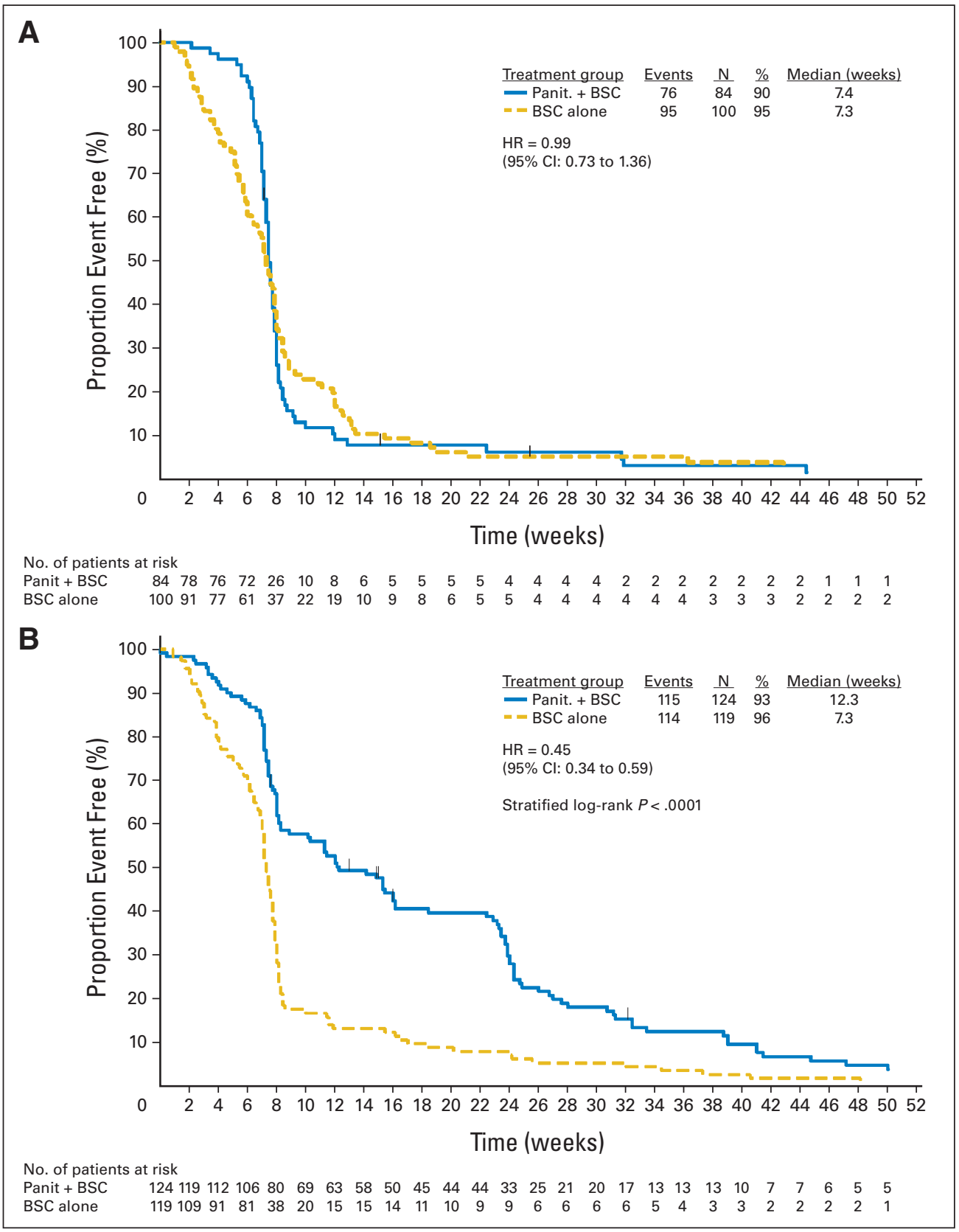

Fig 2. Progression-free survival by treatment within KRAS groups. Progressionfree survival by randomized treatment in (A) mutant and (B) wild-type KRAS groups. Hazard ratios (HR) are shown for panitumumab (panit.) versus best supportive care (BSC) adjusted for randomization factors (Eastern Cooperative Oncology Group score, geographic region). 


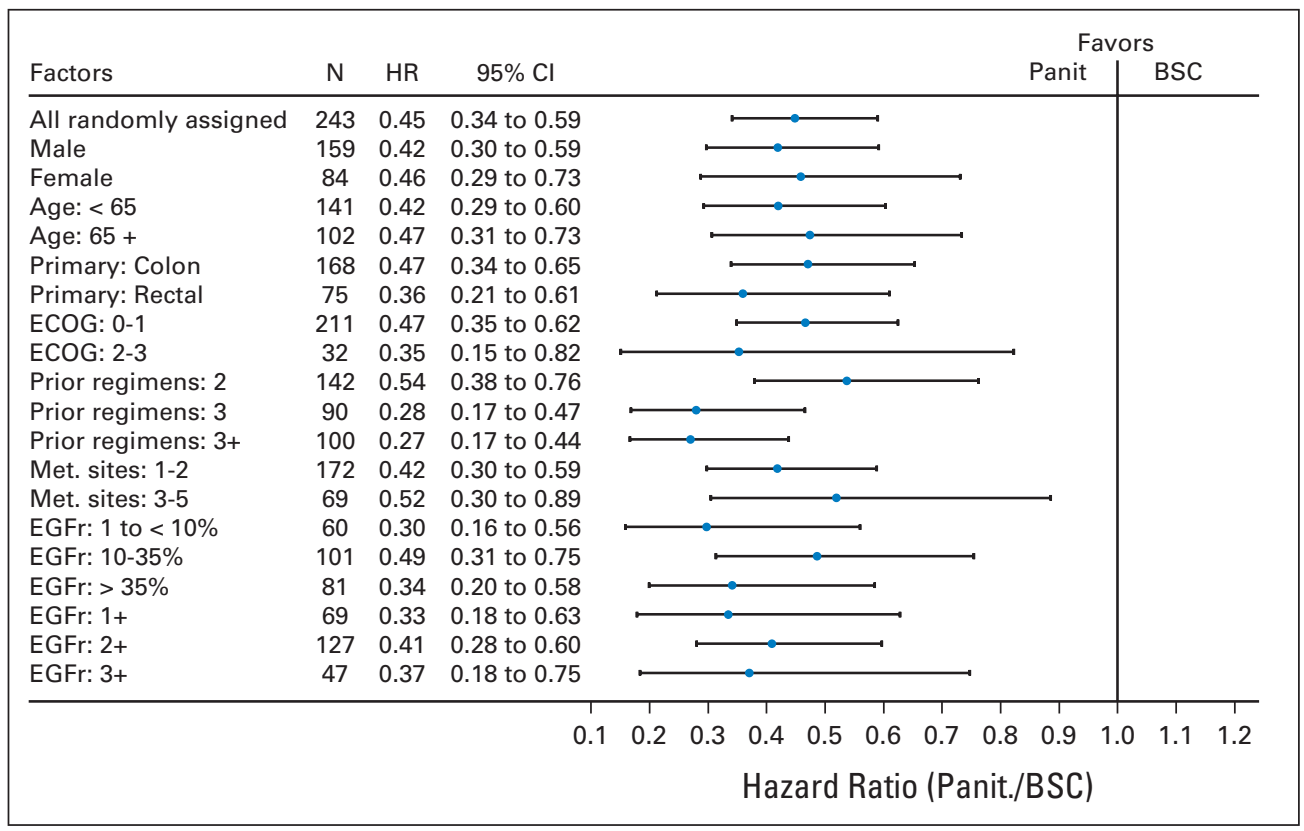

Fig 3. Subset analyses of progressionfree survival in the KRAS wild-type group. Hazard ratio (HR; blue circle) and $95 \% \mathrm{Cl}$ (horizontal lines) adjusted for randomization factors for panitumumab (panit.) versus best supportive care (BSC). N, sample size: HR, hazard ratio; ECOG, Eastern Cooperative Oncology Group; Met, metastatic; EGFr, epidermal growth factor receptor; $1+$, weak; $2+$, moderate; $3+$, strong.

time to cross-over was 7.1 weeks), and, importantly, there was demonstrated benefit of panitumumab after cross-over in patients with WT KRAS tumors. The difference in OS in favor of the WT KRAS group in both treatment arms observed in our study may have reflected a potential prognostic value of KRAS mutational status in CRC or differential sensitivity to panitumumab treatment between KRAS groups.
A

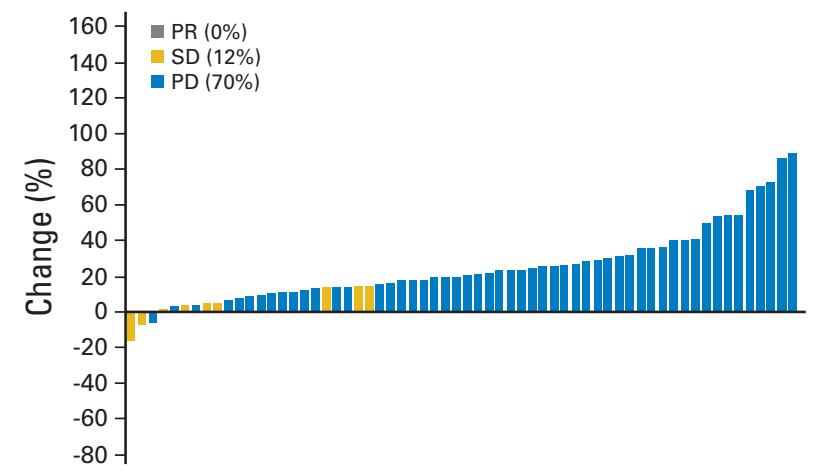

C

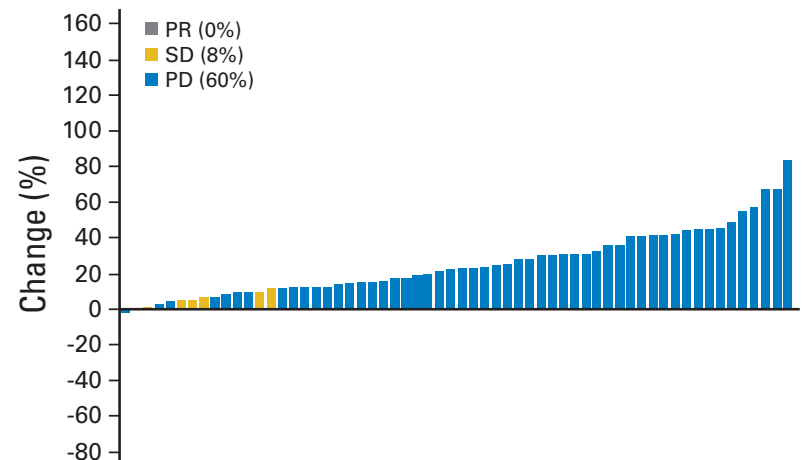

Patient
B

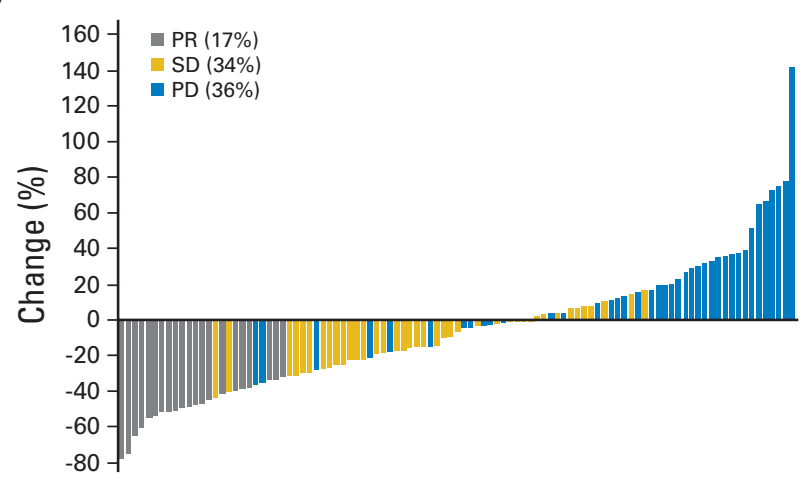

D

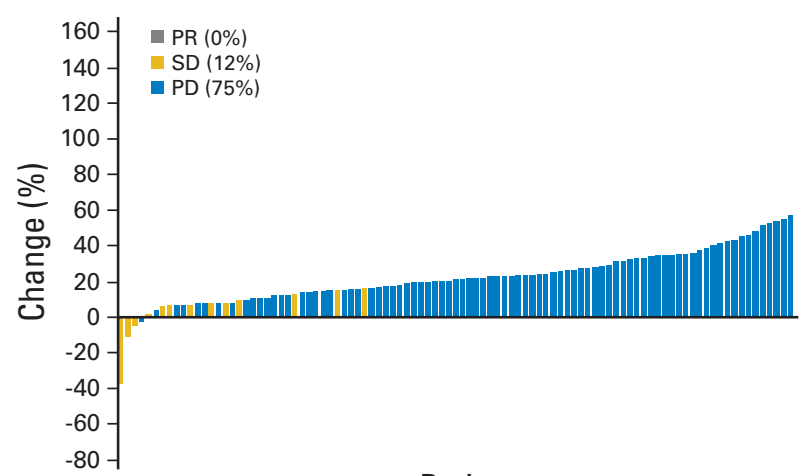

Patient

Fig 4. Waterfall plots showing maximum percent decrease in target lesions (blinded central radiology). (A) Patients receiving panitumumab, mutant $K R A S$. (B) Patients receiving

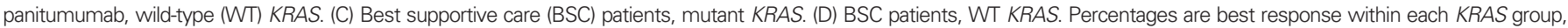
excluding missing or nonassessable postbaseline tumor assessments. PR, partial response (gray); SD, stable disease (yellow); PD, progressive disease (blue). 


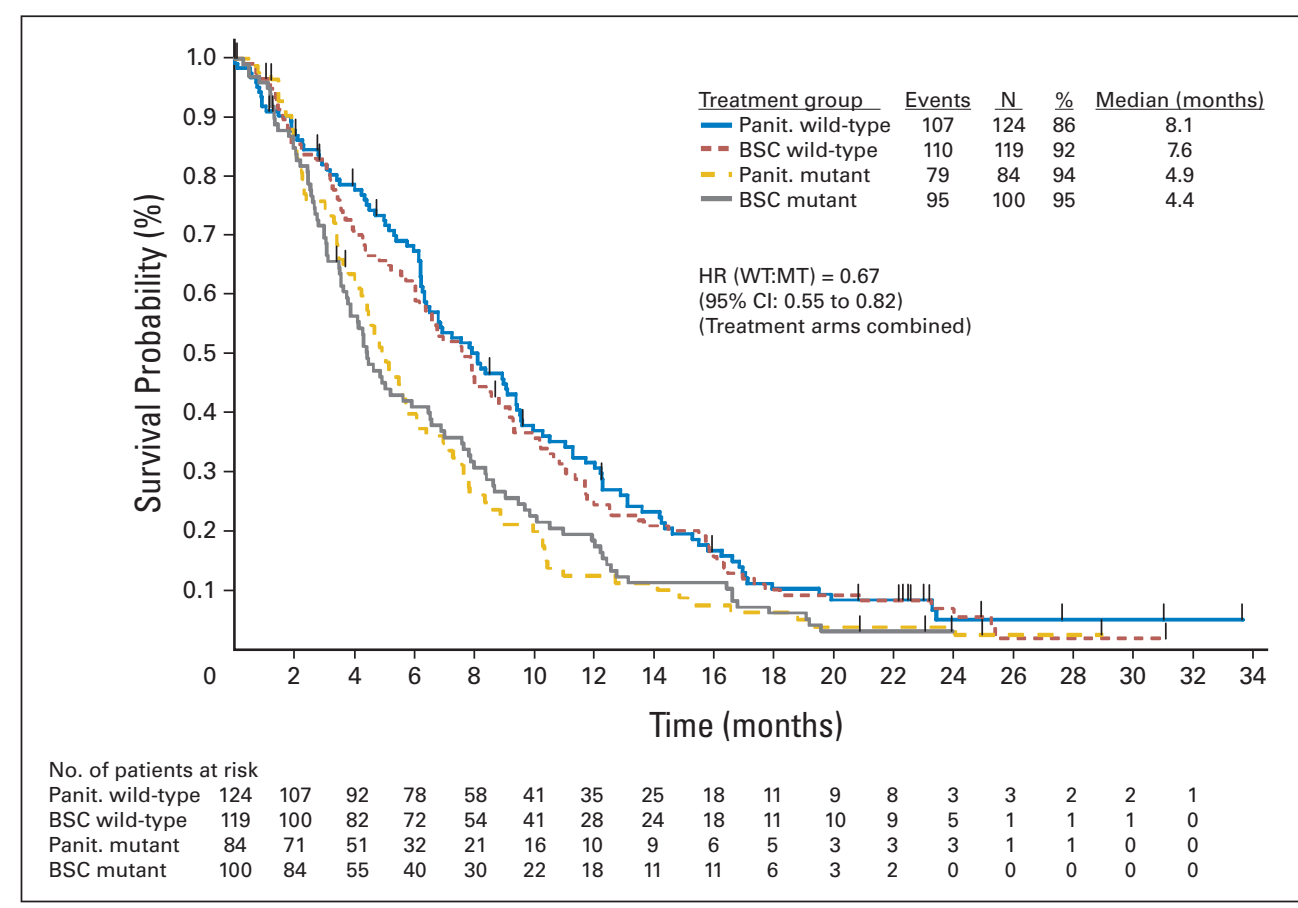

Fig 5. Kaplan-Meier curves for overall survival by treatment and KRAS status. Hazard ratio (HR) for wild-type (WT) versus mutant (MT) KRAS status adjusted for randomized treatment and randomization factors. $\mathrm{Pa}$ nit, panitumumab; BSC, best supportive care; events, deaths; N, sample size.
Although these analyses were conducted retrospectively, several aspects relating to the methodology lend robustness to the results. First, the hypothesis that KRAS mutations may confer primary resistance to anti-EGFR antibodies was generated independently from previous trials. Second, to avoid inflation of type-1 error, samples were only subjected to one biomarker analysis, that of KRAS mutation. Third, the analyses were sufficiently powered and prespecified in a statistical analysis plan before knowledge of KRAS outcome. Fourth, testing was performed by an independent laboratory without patientlevel knowledge of randomization or clinical outcomes. Fifth, the magnitude of the interaction observed is substantial. These considerations, together with consistency with previous studies, and the recognized biologic plausibility of the hypothesis, strongly support the validity of our results and conclusions.

To our knowledge, these are the first results arising from a randomized, controlled trial showing that the state of a signaling molecule downstream of a target plays a crucial role in predicting clinical benefit to a targeted therapeutic. These results also illustrate that the presence of a therapeutic target in itself may be insufficient to predict response to therapy in tumors with multiple molecular alterations. The high positive predictive value (100\% for lack of objective response rate) for mutant KRAS suggests that inhibition of the RAS/RAF/MAPK signaling pathway is primarily responsible for the clinical activity of panitumumab in metastatic $\mathrm{CRC}$, and raises the possibility that mutant KRAS may be predictive in other tumor types. Indeed, EGFR inhibitors have shown modest or no activity in pancreatic cancer, a disease with a high prevalence of KRAS mutations, ${ }^{4,27}$ and in patients with lung cancer whose tumors harbor KRAS mutations. ${ }^{22,28}$

In our study, WT KRAS status was shown to be required but not sufficient to confer sensitivity to panitumumab monotherapy. The mechanisms of primary and treatment-emergent resistance to panitumumab in patients with WT KRAS tumors are unknown.
With regard to primary resistance, EGFR may not be a dominant oncogenic pathway in some tumors, regardless of KRAS status. In addition, while KRAS mutations occur early in the development of CRC, ${ }^{29-31}$ they may also be subsequently acquired, leading to tumor cell heterogeneity. Moreover, while the assay employed in our study is known to detect more than $90 \%$ of known activating KRAS mutations in CRC, it would have missed additional mutations in codons 12 and 61. Other potential mechanisms of resistance include activation of additional tyrosine kinase receptors, such as vascular endothelial growth factor receptor, platelet-derived growth factor receptor, and insulin-like growth factor 1 receptor $^{7}$; activating mutations of additional signaling proteins downstream of the EGFR, such as PI3K, ${ }^{33}$ and $\mathrm{Src},{ }^{34}$ or downstream of KRAS such as $\mathrm{RAF}^{15,35}$; and loss-of-function mutations of tumorsuppressor genes such as phosphatase and tensin homolog $(P T E N) .{ }^{33}$ Elucidating mechanisms of resistance to panitumumab will prove important for the selection of therapeutic combinations to maximize clinical benefit. In addition to ascertaining resistance mechanisms, other biomarkers such as EGFR gene copy number and expression levels of EGFR ligands in tumor cells may be useful to further refine the responder population. ${ }^{32,36}$

The current results apply to the setting of panitumumab monotherapy and indicate that KRAS status should be considered when selecting mCRC patients as candidates for this treatment. Studies are currently underway to assess prospectively whether KRAS mutations also influence response to panitumumab in combination with chemotherapy in earlier lines of therapy. In addition to the relevance of these results to the current use and to the future development of anti-EGFR antibodies, these findings may have implications for the development of oncology therapeutics directed against other targets known to signal though the RAS/RAF/ MAPK pathway. ${ }^{37,38}$ 


\section{AUTHORS' DISCLOSURES OF POTENTIAL CONFLICTS} OF INTEREST

Although all authors completed the disclosure declaration, the following author(s) indicated a financial or other interest that is relevant to the subject matter under consideration in this article. Certain relationships marked with a "U" are those for which no compensation was received; those relationships marked with a " $C$ " were compensated. For a detailed description of the disclosure categories, or for more information about ASCO's conflict of interest policy, please refer to the Author Disclosure Declaration and the Disclosures of Potential Conflicts of Interest section in Information for Contributors.

Employment or Leadership Position: Rafael G. Amado, Amgen Inc (C); Michael Wolf, Amgen Inc (C); Daniel J. Freeman, Amgen Inc (C); Todd Juan, Amgen Inc (C); Robert Sikorski, Amgen Inc (C); Sid Suggs, Amgen Inc (C); Robert Radinsky, Amgen Inc (C); Scott D. Patterson, Amgen Inc (C); David D. Chang, Amgen Inc (C) Consultant or Advisory Role: Marc Peeters, Amgen Inc (C); Eric Van Cutsem, Amgen Inc (U), Merck (U) Stock Ownership: Rafael G. Amado, Amgen Inc; Michael Wolf, Amgen Inc, Dendreon Corp, YM BioSciences Inc; Daniel J. Freeman, Amgen Inc; Todd Juan, Amgen Inc; Robert Sikorski, Amgen Inc; Sid Suggs, Amgen Inc; Robert Radinsky, Amgen Inc; Scott D. Patterson, Amgen Inc; David D. Chang, Amgen Inc Honoraria: Marc Peeters, Amgen Inc Research Funding: Eric Van Cutsem, Amgen Inc, Merck
Expert Testimony: Marc Peeters, Amgen Inc (C) Other Remuneration: Marc Peeters, Amgen Inc

\section{AUTHOR CONTRIBUTIONS}

Conception and design: Rafael G. Amado, Michael Wolf, Salvatore Siena, Daniel J. Freeman, Todd Juan, Robert Sikorski, Robert Radinsky, Scott D. Patterson, David D. Chang

Administrative support: Rafael G. Amado, Robert Radinsky, Scott D. Patterson, David D. Chang

Provision of study materials or patients: Marc Peeters, Eric Van

Cutsem, Salvatore Siena, Sid Suggs

Collection and assembly of data: Rafael G. Amado, Robert Sikorski, Sid Suggs

Data analysis and interpretation: Rafael G. Amado, Michael Wolf, Marc Peeters, Eric Van Cutsem, Salvatore Siena, Daniel J. Freeman, Todd Juan, David D. Chang

Manuscript writing: Rafael G. Amado, Michael Wolf, Daniel J. Freeman, David D. Chang

Final approval of manuscript: Rafael G. Amado, Michael Wolf, Marc

Peeters, Eric Van Cutsem, Salvatore Siena, Daniel J. Freeman, Todd Juan, Robert Sikorski, Sid Suggs, Robert Radinsky, Scott D. Patterson,

David D. Chang

\section{REFERENCES}

1. Shepherd FA, Rodrigues Pereira J, Cialeanu $T$, et al: Erlotinib in previously treated nonsmall-cell lung cancer. N Engl J Med 353:123-132, 2005

2. Cunningham $D$, Humblet $Y$, Siena $S$, et al: Cetuximab monotherapy and cetuximab plus irinotecan in irinotecan-refractory metastatic colorectal cancer. N Engl J Med 351:337-345, 2004

3. Van Cutsem E, Peeters $M$, Siena $S$, et al: Open-label phase III trial of panitumumab plus best supportive care compared with best supportive care alone in patients with chemotherapy-refractory metastatic colorectal cancer. J Clin Oncol 25:1658-1664, 2007

4. Moore MJ, Goldstein D, Hamm J, et al: Erlotinib plus gemcitabine compared with gemcitabine alone in patients with advanced pancreatic cancer: A phase III trial of the National Cancer Institute of Canada Clinical Trials Group. J Clin Oncol 25: 1960-1966, 2007

5. Mendelsohn J, Baselga J: Epidermal growth factor receptor targeting in cancer. Semin Oncol 33:369-385, 2006

6. Hynes NE, Lane HA: ERBB receptors and cancer: The complexity of targeted inhibitors. Nat Rev Cancer 5:341-354, 2005

7. Adams R, Maughan T: Predicting response to epidermal growth factor receptor-targeted therapy in colorectal cancer. Expert Rev Anticancer Ther 7:503-518, 2007

8. Chung KY, Shia J, Kemeny NE, et al: Cetuximab shows activity in colorectal cancer patients with tumors that do not express the epidermal growth factor receptor by immunohistochemistry. J Clin Oncol 23:1803-1810, 2005

9. Mitchell EP, Hecht JR, Baranda J, et al: Panitumumab activity in metastatic colorectal cancer (mCRC) patients (pts) with low or negative tumor epidermal growth factor receptor (EGFr) levels: An updated analysis. J Clin Oncol 25:184s, 2007 (suppl; abstr 4082)

10. Downward J: Targeting RAS signalling pathways in cancer therapy. Nat Rev Cancer 3:11-22, 2003

11. Schubbert S, Shannon K, Bollag G: Hyperactive Ras in developmental disorders and cancer. Nat Rev Cancer 7:295-308, 2007

12. Bos JL: Ras oncogenes in human cancer: $A$ review. Cancer Res 49:4682-4689, 1989

13. Malumbres $M$, Barbacid $M$ : RAS oncogenes: The first 30 years. Nat Rev Cancer 3:459465, 2003

14. Andreyev HJ, Norman AR, Cunningham $D$, et al: Kirsten ras mutations in patients with colorectal cancer: The 'RASCAL II' study. Br J Cancer 85:692696, 2001

15. Benvenuti S, Sartore-Bianchi A, Di Nicolantonio $F$, et al: Oncogenic activation of the RAS/RAF signaling pathway impairs the response of metastatic colorectal cancers to anti-epidermal growth factor receptor antibody therapies. Cancer Res 67: 2643-2648, 2007

16. Di Fiore F, Blanchard F, Charbonnier F, et al: Clinical relevance of KRAS mutation detection in metastatic colorectal cancer treated by cetuximab plus chemotherapy. Br J Cancer 96:1166-1169, 2007

17. Esteller M, Gonzalez S, Risques RA, et al: K-ras and p16 aberrations confer poor prognosis in human colorectal cancer. J Clin Oncol 19:299-304, 2001

18. Ince WL, Jubb AM, Holden SN, et al: Association of k-ras, b-raf, and p53 status with the treatment effect of bevacizumab. J Natl Cancer Inst 97:981-989, 2005

19. Lievre A, Bachet JB, Le Corre $D$, et al: KRAS mutation status is predictive of response to cetuximab therapy in colorectal cancer. Cancer Res 66: 3992-3995, 2006

20. Bazan V, Migliavacca M, Zanna I, et al: Specific codon $13 \mathrm{~K}$-ras mutations are predictive of clinical outcome in colorectal cancer patients, whereas codon $12 \mathrm{~K}$-ras mutations are associated with mucinous histotype. Ann Oncol 13:1438-1446, 2002

21. De Roock W, Piessevaux H, De Schutter J, et al: KRAS wild-type state predicts survival and is associated to early radiological response in metastatic colorectal cancer treated with cetuximab. Ann Oncol [epub ahead of print on November 12, 2007]

22. Pao W, Wang TY, Riely GJ, et al: KRAS mutations and primary resistance of lung adenocarcinomas to gefitinib or erlotinib. PLoS Med 2:e17, 2005

23. Newton CR, Graham A, Heptinstall LE, et al: Analysis of any point mutation in DNA: The amplification refractory mutation system (ARMS). Nucleic Acids Res 17:2503-2516, 1989

24. Thelwell N, Millington S, Solinas A, et al: Mode of action and application of scorpion primers to mutation detection. Nucleic Acids Res 28:37523761,2000

25. Whitcombe D, Theaker J, Guy SP, et al: Detection of PCR products using self-probing amplicons and fluorescence. Nat Biotechnol 17:804-807, 1999

26. Gail M, Simon R: Testing for qualitative interactions between treatment effects and patient subsets. Biometrics 41:361-372, 1985

27. Philip PA, Benedetti J, Fenoglio-Preiser C, et al: Phase III study of gemcitabine [G] plus cetuximab [C] versus gemcitabine in patients (pts) with locally advanced or metastatic pancreatic adenocarcinoma [PC]: SWOG S0205 study. J Clin Oncol 25:18s, 2007 (abstr 4509)

28. Massarelli E, Varella-Garcia M, Tang $X$, et al: KRAS mutation is an important predictor of resistance to therapy with epidermal growth factor receptor tyrosine kinase inhibitors in non-smallcell lung cancer. Clin Cancer Res 13:2890-2896, 2007

29. Vogelstein B, Fearon ER, Hamilton SR, et al: Genetic alterations during colorectal-tumor development. N Engl J Med 319:525-532, 1988

30. Burmer GC, Loeb LA: Mutations in the KRAS2 oncogene during progressive stages of human colon carcinoma. Proc Natl Acad Sci U S A 86:2403-2407, 1989 
31. Hasegawa $H$, Ueda $M$, Watanabe $M$, et al: $\mathrm{K}$-ras gene mutations in early colorectal cancer: Flat elevated vs polyp-forming cancer. Oncogene 10: 1413-1416, 1995

32. Khambata-Ford S, Garrett CR, Meropol NJ, et al: Expression of epiregulin and amphiregulin and K-ras mutation status predict disease control in metastatic colorectal cancer patients treated with cetuximab. J Clin Oncol 25:3230-3237, 2007

33. Nahta $R, Y u D$, Hung $M C$, et al: Mechanisms of disease: Understanding resistance to HER2- targeted therapy in human breast cancer. Nat Clin Pract Oncol 3:269-280, 2006

34. Lu $Y$, Li $X$, Liang $K$, et al: Epidermal growth factor receptor (EGFR) ubiquitination as a mechanism of acquired resistance escaping treatment by the anti-EGFR monoclonal antibody cetuximab. Cancer Res 67:8240-8247, 2007

35. Davies H, Bignell GR, Cox C, et al: Mutations of the BRAF gene in human cancer. Nature 417:949954,2002
36. Sartore-Bianchi A, Moroni M, Veronese S, et al: Epidermal growth factor receptor gene copy number and clinical outcome of metastatic colorectal cancer treated with panitumumab. J Clin Oncol 25:3238-3245, 2007

37. Birchmeier C, Birchmeier W, Gherardi E, et al: Met, metastasis, motility and more. Nat Rev Mol Cell Biol 4:915-925, 2003

38. Pollak MN, Schernhammer ES, Hankinson SE: Insulin-like growth factors and neoplasia. Nat Rev Cancer 4:505-518, 2004

\section{Acknowledgment}

We thank the patients, families, and the study staffs for study participation, and the following individuals from Amgen Inc: C. Tucknott and P. D’Avirro (study management); A. Rong (statistical planning and analyses); A. Bakker and S. Ildiko (sample management/assay coordination); and L. Runft (writing assistance). The ClinicalTrials.gov identifier numbers for studies 20020408 and 20030194 are NCT00113763 and NCT00113776, respectively.

\section{Appendix}

The Appendix is included in the full-text version of this article, available online at www.jco.org. It is not included in the PDF version

(via Adobe ${ }^{\circledR}$ Reader $^{\circledR}$ ). 